\title{
Nano-Forensics a Comprehensive Review
}

ISSN: 2578-0042

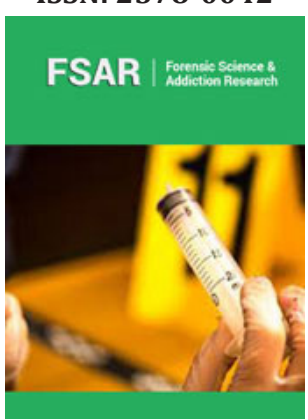

*Corresponding author: Paikrao Hariprasad, Assistant Professor, Department of Forensic Biology, Government Institute of Forensic Science, Nagpur, India

Submission: 眥January 05, 2021

Published: 悳 February 03, 2021

Volume 5 - Issue 3

How to cite this article: Paikrao Hariprasad, Dipale Ashlesha, Patil Anita, Tajane Diksha. Nano-Forensics a Comprehensive Review. Forensic Sci Add Res. 5(3). FSAR. 000618. 2021. DOI: 10.31031/FSAR.2021.05.000618

Copyright@ Paikrao Hariprasad, This article is distributed under the terms of the Creative Commons Attribution 4.0 International License, which permits unrestricted use and redistribution provided that the original author and source are credited.

\section{Paikrao Hariprasad ${ }^{1 *}$, Dipale Ashlesha ${ }^{2}$, Patil Anita ${ }^{3}$ and Tajane Diksha ${ }^{4}$}

${ }^{1}$ Assistant Professor, Department of Forensic Biology, Government Institute of Forensic Science, Nagpur, India

${ }^{2}$ Post graduate student, Department of Forensic Biology, Government Institute of Forensic Science, Nagpur, India

${ }^{3}$ Professor, Department of Biotechnology, Sant Gadge Baba Amravati University Amravati, India

${ }^{4}$ Research Scholar, NAU, Navsari, India

\begin{abstract}
Nanotechnology is becoming popular in today's world, with the nano-revolution touching almost in every field. It offers a promising application in electronics, diagnosis, biosensing, drug delivery, and imaging. Nano-Forensic is emerging as a novel discipline in the field of forensic science. Nanotechnology has been utilized extensively in forensic science to detect and analyse evidence at a nanoscale level. Nano analysis is transforming the investigation process by making them more accurate, faster, and more sensitive. As nanomaterials have the property of enhancing the detection limit at nanoscale level it has been widely used to detect fingerprint, explosives, unlawful drugs, toxic substances and DNA samples.
\end{abstract}

Keywords: Nano-sensors; Forensic science; Nano-forensics; Nano-PCR

\section{Introduction}

Nanotechnology is making a valuable contribution in every field. It is a widely used technique because of its ability to manipulate and characterize the matter at a level of single atoms and small atoms [1]. The word "nano" is derived from the ancient Greek "Nanos," meaning "dwarf," which refers to the "billionth" or a factor of 10-9. In general, to understand $1 \mathrm{~nm}$ is about 3-10 atoms wide. It is very tiny when compared with the standard size encountered day-to-day. E.g., $1 \mathrm{~nm}$ is $1 / 1000$ th the width of human hair. Nanotechnology described the study aspect concerning the science, engineering, and technology conducted at a scale that ranges between 1 to 100 nanometers [2]. The potential of nanotechnology in electronics, diagnostics, biosensing, imaging, optical devices, and drug delivery due to their small size, large surface area, and enhanced reactivity [3-7]. Its multi-purpose application in almost every field has made it a universal purpose technology [2]. Therefore this universal technology has a plentiful of applications in the field of forensic science. Nano-Forensic, an exquisite blend of nanotechnology and forensic science, is entirely a novel discipline in forensic science. Nano-Forensic helps identify and examine evidence at the nanoscale level as earlier, it is impossible to analyze the critical evidence because of the instrument's detection limit. With advancements in the techniques, Nano analysis is transforming the investigation process by making them more accurate, faster, and more sensitive $[8,9]$. Nano forensic and other technologies have significant fingerprint analysis applications, explosive detection, drug screening, toxic substance analysis, and DNA analysis $[10,11]$. This review would briefly update how nanotechnology is widely used in forensic analysis of evidence.

\section{Nanomaterials for latent fingerprint analysis}

Nanoparticles have been used for decades to develop the latent fingerprint, with silver nanoparticles being used from 1970. The small size of nanoparticles allows them to bind with the minute ridges of a fingerprint, further enhancing the ridge details on fingerprints and 
sweat pores. Morris presented that silver nanoparticles can be used as physical developers to visualized latent fingerprints on paper [12]. During the reaction, the silver nanoparticles formed with the organic constituent of fingerprints, which develop the fingerprint in dark grey or black sliver image on the porous surface [13]. Gold nanoparticles are beneficial for latent fingerprint analysis as they are inert, highly selective, and sensitive. It has the advantage that latent print produced by gold nanoparticles can be store for a longer duration. Gold nanoparticles are used to improve latent fingerprints' visibility by multi-metal deposition (MMD) and single metal deposition due to these properties $[14,15]$.

Quantum Dots and fluorescent materials have gained significant attention due to their small size and excellent fluorescent intensity. A study carried out by Dr. Roland Menzel [16] shows that Quantum Dots can visualize [16]. Quantum dots can also be used for the development of bloody fingerprints. Bloody fingerprints have high chances of smearing and contamination, which can damage the ridge details in fingerprints. It was overcome by quantum dots having the fluorescence property used to analyze bloody prints [17]. The study shows that incorporating the minor amount of $\mathrm{ZnO}$ SiO2 nanoparticles in powder increases the visual enhancement of latent print to third-level ridge details of prints [18]. Carbon nano powder has been developing for the visualization of prints against multi-colored or patterned backgrounds [17].

\section{Role of nanoparticles in explosives detection}

Explosives-based terrorism has been rising for the last few years. Explosives such as bombs, improvised explosives devices (IEDs), grenades are causing widespread mass destruction [19]. Over the year, various sensing devices have been developed for the detection of trace explosives. Because of nanomaterial's unique electrical and optical properties, it is widely used to develop lowcost sensors. Sensing of the explosives is usually done through biologically based sensors [20]. Immunosensing techniques provide a great sensitivity in detecting the TNT with a detection limit as low as $0.09 \mathrm{~g} / \mathrm{ml}$ [21]. Frances $\mathrm{S}$ et al. [22] prepared a capillary immunosensor for the specific TNT detection by using the AntiTNT antibody [22]. Carbon-based nanomaterials, including carbon nanotubes, graphene, and carbon nanoparticles, have properties like chemically inert, low cytotoxicity, high biocompatibility, and unique electronic properties the applications of these materials as sensors. Chen et al. utilized graphene oxide as an oxidized derivative of graphene to detect nitroaromatic explosives. They constructed the graphene-based sensor and analyzed compounds like dinitro toluene (DNT), dinitrobenzene (DNB), and trinitrobenzene (TNB) [23].

\section{Nanomaterial in DNA analysis}

DNA analysis has a tremendous potential benefit for the civil and criminal justice system [24]. It establishes the identity of an individual in forensic investigation. Nowadays, nanoparticle-based methods influence DNA analysis because of their low cost, easy automation, and convenient operation. Precisely, the magnetic nanoparticles are used to extract DNA because of increased sensitivity and high DNA yield. Nongyue He et al. used the Fe304 nanoparticles to extract nucleic acid from four different sources such as bacteria, yeast, human blood, and virus. The results showed that using magnetic nanoparticles to extract nucleic acid gives the high yield and relatively high purity of nucleic acids [25].

Sensor-based DNA detection technique has applications in DNA analysis. Mostly the gold nanoparticles are used for sensing mechanisms because of their optothermal property. Cheong, et al. [26] used Au nanorods for the extraction of DNA from the cell. Using the optothermal property researchers transformed the near infra-red energy into the thermal energy in a microfluidic chip that results in lysis of pathogen and eventually in the extraction of DNA [26]. Apart from gold nanoparticles, silica nanoparticle-based assay also detects DNA with the great sensitivity of 10pM [27]. The NanoPCR, a nanoparticle assisted PCR, is gaining considerable attention because of its specificity and reaction speed. Various types of nanoparticles like carbon tubes, quantum dots, and metal nanoparticles are introduced into the PCR technology. These nanoparticles are improving the efficiency and specificity of PCR products [28-30].

\section{Nanoparticles in illicit drug analysis}

The demand for illicit drugs is continuously increasing. Cannabis, amphetamine-type substances, cocaine, and heroin continue to be the most prevalent illicit drugs, but comparatively, new psychoactive agents have also been increasing in the market. In criminal investigations, drug analysis is a significant branch of modern analytical chemistry with many legal and socially applicable consequences. With the advancement in technology, sensing devices are used to detect drugs [31]. Sensing using nanoparticles usually takes place through colorimetric, fluorescence, and electrochemical sensors [32,33]. Gandhi, et al. [34] reported the development of dipstick assay based on the AuNP labeled single-chain fragment variable ( $\mathrm{scFv}$ ) antibody for the detection of morphine. The developed dipstick is suitable for analyzing the morphine from different biological fluids like blood, urine, and saliva [34]. Au nanoparticles show a significant Surface Plasmon Resonance phenomenon. It is used in colorimetric sensors. Gao et al. used a colorimetric sensor based on aptamer and molybdenum disulfide (MoS2)-gold nanoparticles (AuNPs) to detect cocaine. This sensor has been found to be rapid, cost-effective, and highly sensitive [35]. Different quantum dots techniques along with the other techniques, are also being used for fluorescence-based sensing because of their excellent quantum yield and fluorescence [36].

\section{Conclusion}

The potential of nanotechnology is making a positive contribution to forensic science to solve the crime. Various types of nanoparticles are in use for the detection of various forensic samples. Nano-sensors applications in Nano-forensics are due to high sensitivity. Further, Nano-Forensics has made the investigation process rapid. Nanotechnology can benefit in the future as an advanced and preventive tool in different field of forensic science. 


\section{References}

1. Wonders S (2002) The importance of nanoscale science and technology Endless frontiers: A review of the national nanotechnology initiative. National Academies Press, Washington, USA.

2. Shukla R, Anand Lodha S, Alok Pandya (2016) Nanotechnology: An applied and robust approach for forensic investigation. Forensic Res Criminol Int J 2(1): 35-37.

3. He Z, Zhang Z, Bi S (2020) Nanoparticles for organic electronics applications. Materials Research Express 7(1): 1-13.

4. Malefane M (2020) Applications of nanotechnology towards detection and treatment of HIV/AIDs: A review article. Res Dev Material Sci 12(5): 1315-1321.

5. Gautam P, Sharma R, Lata K, Rajput Y, Mann B (2017) Construction of a lateral flow strip for detection of soymilk in milk. J Food Sci Technol 54(13): 4213-4219.

6. DiGiovanni D, Li M, Willner A (2013) Fiber optic nanotechnology: A new frontier of fiber optics. Nano photonics 2(5-6): 311-313.

7. Pavan Kumar D, Arati Sharma, Sinisa D, Shantu A, Dhimant D (2020) Nanotherapeutics: A mini review. Mod Appro Drug Des 2(5): 1-3.

8. Vivek C, Vandana S, Archana T (2016) Application of nanotechnology in forensic science. Int J Life Sci Scienti Res 3(3): 1047-1051.

9. Pandya A, Shukla R (2018) New perspective of nanotechnology: Role in preventive forensic. Egyptian Journal of Forensic Sciences 8(1): 1-11.

10. Chen Y (2011) Forensic applications of nanotechnology. Journal of the Chinese Chemical Society 58(6): 828-835.

11. Kaushik M, Mahendru S, Chaudhary S, Kukreti S (2017) DNA fingerprints: Advances in their forensic analysis using nanotechnology. Journal of Forensic Biomechanics 8(1): 1-4.

12. Cantujames A, Johnson L (2001) Silver physical development of latent prints. In: Lee H \& Gaensslen R (Eds.), Advances in fingerprint technology. CRC Press, Boca Raton, Florida, USA.

13. Sodhi G, Kaur J (2016) Physical developer method for detection of latent fingerprints: A review. Egyptian Journal Of Forensic Sciences 6(2): 4447.

14. Gao D, Li F, Song J, Xu X, Zhang Q et al. (2009) One step to detect the latent fingermarks with gold nanoparticles. Talanta 80(2): 479-483.

15. Mohamed A (2011) Gold is going forensic. Gold Bulletin 44(2): 71-77.

16. Menzel E (2001) Fingerprint detection with photoluminescent nanoparticles. In: Lee H, Gaensslen R (Eds.), Advances in fingerprint technology. CRC Press, Boca Raton, Florida, USA

17. Becue A, Moret S, Champod C, Margot P (2009) Use of quantum dots in aqueous solution to detect blood fingermarks on non-porous surfaces. Forensic Sci Int 191(1-3): 36-41.

18. Arshad A, Farrukh M, Ali S, Khaleeq M, Tahir M (2015) Development of latent fingermarks on various surfaces using $\mathrm{ZnO}_{-} \mathrm{SiO}_{2}$ nanopowder. J Forensic Sci 60(5): 1182-1187.

19. Rawtani D, Tharmavaram M, Pandey G, Hussain C (2019) Functionalized nanomaterial for forensic sample analysis. TrAC Trends in Analytical Chemistry 120: 115661.
20. Smith R, D Souza N, Nicklin S (2008) A review of biosensors and biologically inspired systems for explosives detection. Analyst 133(5): 571-584.

21. Shankaran D, Gobi K, Sakai T, Matsumoto K, Imato T, et al. (2005) A novel surface plasmon resonance immunosensor for 2, 4, 6-trinitrotoluene (TNT) based on indirect competitive immunoreaction: A promising approach for on-site landmine detection. IEEE Sensors Journal 5(4): 616-621.

22. Narang U, Gauger P, Ligler F (1997) A displacement flow immunosensor for explosive detection using microcapillaries. Anal Chem 69(14): 27792785 .

23. Chen TW, Sheng ZH, Wang K, Wang FB, Xia XH (2011) Determination of explosives using electrochemically reduced graphene. Chemistry-An Asian Journal 6(5): 1210-1216.

24. National Acad Press (1992) DNA technology in forensic science. Washington, DC, USA.

25. Ma C, Li C, Wang F, Ma N, Li X, et al. (2013) Magnetic nanoparticles-based extraction and verification of nucleic acids from different sources. J Biomed Nanotechnol 9(4): 703-709.

26. Cheong KH, Yi D, Lee J, Park J, Kim M, et al. (2008) Gold nanoparticles for one step DNA extraction and real-time PCR of pathogens in a single chamber. Lab Chip 8(5): 810-813.

27. Wang Y, Liu B (2007) Silica nanoparticle assisted DNA assays for optical signal amplification of conjugated polymer based fluorescent sensors. Chem Commun 34: 3553-3555.

28. Ma L, He S, Huang J, Cao L, Yang F, et al. (2009) Maximizing specificity and yield of PCR by the quantum dot itself rather than property of the quantum dot surface. Biochimie 91(8): 969-973.

29. Li H, Huang J, Lv J, An H, Zhang X, et al. (2005) Nanoparticle PCR: Nanogold-assisted PCR with enhanced specificity. Angew Chem Int Ed Engl 44(32): 5100-5103.

30. Cui D, Tian F, Kong Y, Titushikin I, Gao H (2003) Effects of single-walled carbon nanotubes on the polymerase chain reaction. Nanotechnology 15(1): 154 .

31. Lad A, Pandya A, Agrawal Y (2016) Overview of nano-enabled screening of drug-facilitated crime: A promising tool in forensic investigation. TrAC Trends In Analytical Chemistry 80: 458-470.

32. Chen C, Wang C, Ko P, Chen Y (2020) Nanomaterial-based adsorbents and optical sensors for illicit drug analysis. Journal of Food and Drug Analysis 28(4): 655-677.

33. Majeed M, Nawaz H, Arshad F (2020) Detecting the presence of illicit drugs using biosensors. Nanobiosensors: From Design to Applications, pp. 223-253.

34. Gandhi S, Banga I, Maurya P, Eremin S (2018) A gold nanoparticlesingle-chain fragment variable antibody as an immunoprobe for rapid detection of morphine by dipstick. RSC advances 8(3): 1511-1518.

35. Gao L, Xiang W, Deng Z, Shi K, Wang H, et al. (2020) Cocaine detection using aptamer and molybdenum disulfide-gold nanoparticle-based sensors. Nanomedicine 15(4): 325-335.

36. Farahani M, Khademabbasi K, Mollatayefeh N (2018) A selective morphine nanosensor derived from functionalized CdS quantum dots. Materials Letters 228: 68-71. 